\title{
On a continuous transfer of history variables for frictional contact problems based on interpretations of covariant derivatives as a parallel translation
}

Alexander Konyukhov, Karl Schweizerhof

Universität Karlsruhe, Institut für Mechanik

Institut für Mechanik

Kaiserstr. 12, Geb. 20.30

76128 Karlsruhe

Tel.: +49 (0) 721/ 608-2071

Fax: +49 (0) 721/ 608-7990

E-Mail: ifm@uni-karlsruhe.de

www.ifm.uni-karlsruhe.de 


\title{
On a continuous transfer of history variables for frictional contact problems based on interpretations of covariant derivatives as a parallel translation
}

\author{
Alexander Konyukhov, Karl Schweizerhof
}

2006

\section{Abstract}

Regularization methods based on the penalization of the tangent displacements are among the most exploited techniques in combination with finite element methods to model frictional interactions. Usually the global tangent displacements are described via convective coordinates which are e.g. used in a finite element discretization of the contact surface. These displacements serve to compute the tangent tractions in the case of sticking via a regularization procedure as well as in the case of sliding via a return-mapping scheme. The convective coordinates of the contact point as well as the corresponding tangent tractions are considered as history variables and have to be stored for each load step. In this contribution, we discuss the particular issue of continuous transfer for history variables in the case of large deformation problems adapted for the covariant contact description developed in Konyukhov and Schweizerhof [4]. Some specific examples are chosen to illustrate the effect of incorrect transfer for both non-frictional and frictional problems and, therefore, the necessity of the proposed techniques.

\section{Introduction}

The formulation of the frictional contact conditions in rate form is an essential part in the development of efficient algorithms. Several approaches are known in literature [6], [10]: these are a) the Lagrangian multiplier method, b) various regularization techniques such as the penalty method and the augmented Lagrangian method, and c) combined methods such as Nitsches method. In the general case of large deformation contact problems, contacting bodies are meshed with dissimilar meshes. The necessary information about contact interactions such as contact tractions for both sticking and sliding cases, and sliding displacements are assigned to contact pairs, e.g., to nodal contact pairs within the node-to-node approach, the latter of course only in the case of small deformation. Separate assignments either to a master segment, or to a slave segment 
are normally chosen for large displacement problems, mainly due to robustness reasons. Thus, a problem of information passing, or, so-called, the problem of transfer of history variables between master and slave segments, neighboring masters segments or neighboring slave segments occurs. Of course, this transfer has to be done in accordance with both the equilibrium conditions on the contact surface, and the corresponding interface conditions. The first conditions are normally directly fulfilled in contact mechanics, while in fluid-structure interaction problems, they are treated separately known as conservative load transfer conditions, see [2]. The second, interface conditions include the treatment of a constitutive law defined between surfaces, e.g., the Coulomb friction law, together with a proper numerical scheme, such as the return-mapping scheme.

\section{Transfer algorithm for a trial force}

The description in convective variables has been introduced in contact mechanics for careful treatment of frictional contact problems in particular to avoid artefacts due to improper discretization [7]. The tangent surface displacements are described via convective variables and, in due course, they are used to compute the tangent tractions in the case of sticking via a regularization procedure as well as in the case of sliding via a return-mapping scheme. Within the master-slave approach, the convective coordinates are defined, of course, on the master side, while the information about the contact forces should be supplied for both, the master and the slave sides. Problems arise, when a slave segment is crossing several master segments during deformation, see Fig. 1.

Let us assume, that at step $(n)$ a slave point $S^{(n)}$ is projected onto the master segment $\mathbf{A}^{(n)}$, giving a contact point $C^{(n)}$, defined via convective coordinates $\xi_{A}^{1}, \xi_{A}^{2}$. At load step $(n+1)$, the same slave point is moved into position $S^{(n+1)}$ and projected onto the neighboring master segment $\mathbf{B}^{(n+1)}$ giving a contact point $\mathbf{C}^{(n+1)}$, defined via convective coordinates $\xi_{B}^{1}, \xi_{B}^{2}$. Since the convective coordinates $\xi_{A}^{1}, \xi_{A}^{2}$, resp. $\xi_{B}^{1}, \xi_{B}^{2}$ are defined only locally on each contact element, a problem concerning the correct transfer of history variables while crossing the element boundaries arises for large displacement problems. A typical example is the computation of components for a trial tangent force

$$
T_{i}^{(n+1)}=T_{i}^{(n)}-\epsilon_{T} a_{i j} \Delta \xi^{j}
$$

leading to a maximum jump in tangent forces as $2 \epsilon_{T}$, see details in [5].

Various approaches can be exploited to solve this problem. Wriggers et. al. [9] proposed an algorithm for the 2D case, based on the usage of the path length of the projection point $C$. In this case the full geometrical information about the 


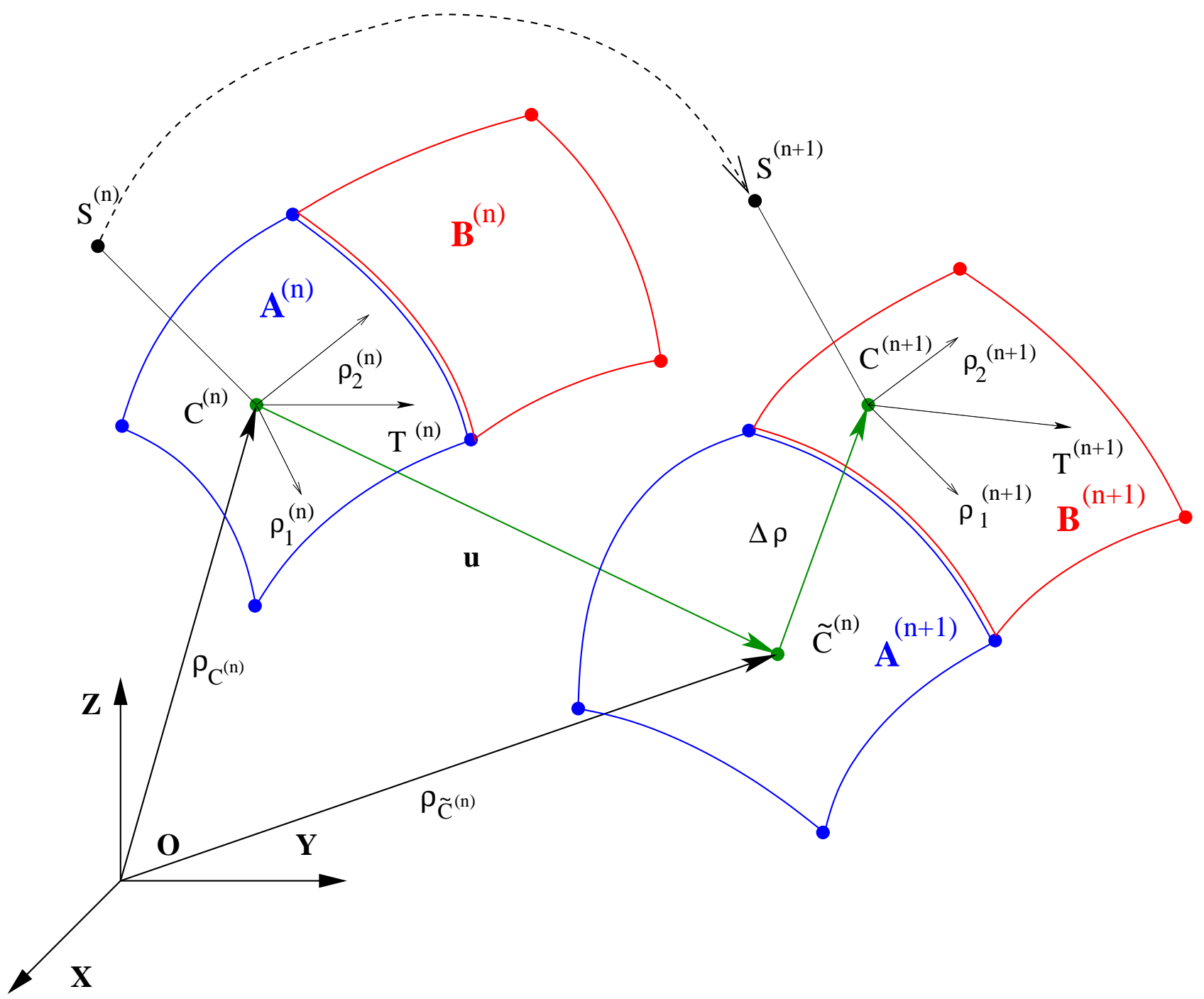

Figure 1: Contact point moving across element boundaries. Covariant derivatives. Sketch of integration scheme.

path passed by the slave point has to be stored. Puso and Laursen [8] proposed to determine increments of convective coordinates in the geometric form for the 3D case. In this case 3D coordinates of slave nodes are stored, but the geometry of the contact segments is no longer captured. As an alternative we consider a continuous transfer algorithm of first order with respect to convective coordinates based upon the interpretation of covariant derivatives as a parallel translation. A pair of points $S^{(n)}$ and $C^{(n)}$ defines then a spatial coordinate system corresponding to the closest point projection. For the terminology and detailed information we refer to the covariant description given in [4]. The following steps lead to a transfer algorithm for trial forces:

a) The tangent components of a traction vector $T^{(n)}$ on the master segment $\mathbf{A}^{(n)}$ have to be transformed in tensor form into the tangent components on the master segment $\mathbf{B}^{(n)}$. 
b) The increment vector $\Delta \boldsymbol{\rho}$ has to be expressed in the surface metrics of the current contact segment $\mathbf{B}^{(n+1)}$.

The increment vector $\Delta \boldsymbol{\rho}$ is obtained in the global reference Cartesian system as

$$
\Delta \boldsymbol{\rho}=\left.\boldsymbol{\rho}_{C^{(n+1)}}\right|_{\xi_{A}^{1}, \xi_{A}^{2}}-\left.\left(\boldsymbol{\rho}_{C^{(n)}}+\mathbf{u}_{C^{(n)}}\right)\right|_{\xi_{B}^{1}, \xi_{B}^{2}},
$$

where the first term is representing the current position of the contact point $C^{(n+1)}$, while the terms in brackets can be recomputed knowing only two previous convective coordinates $\xi_{A}^{1}, \xi_{A}^{2}$ as well as the previous segment A. According to the aforementioned steps, the continuous analog of a trial step in eqn. (1) is build as follows

$$
T_{B,(n+1)}^{j}=T_{A,(n)}^{i}\left(\boldsymbol{\rho}_{i}^{A} \cdot \boldsymbol{\rho}_{B}^{j}\right)-\epsilon_{T}\left(\Delta \boldsymbol{\rho} \cdot \boldsymbol{\rho}_{B}^{j}\right),
$$

where $\boldsymbol{\rho}_{B}^{j}$ are current contravariant basis vectors computed for segment $\mathbf{B}$ at a point $\xi_{B}^{1}, \xi_{B}^{2} ; \boldsymbol{\rho}_{i}^{A}$ are covariant basis vectors computed for the previous segment A at a point $\xi_{A}^{1}, \xi_{A}^{2}$; the tangent traction vector components $T^{j}$ are taken in contravariant form for both current and previous segments. The standard return mapping algorithm for the Coulomb friction is applied then to compute the real frictional force.

The update algorithm for sliding displacements, see details in [5], has to be adjusted according to the continuous transfer algorithm, namely, the new coordinates of the elastic region have to be recomputed with new sliding displacements as

$$
\Delta \xi_{s l}^{i}=\left(\Delta \boldsymbol{\rho}_{s l} \cdot \boldsymbol{\rho}_{B}^{j}\right)
$$

where $\Delta \boldsymbol{\rho}_{s l}$ is computed in analogy to eqn. (2), but in the center of the elastic region $\xi_{s l}^{1}, \xi_{s l}^{2}$.

The algorithm is straightforward if history variables are stored at the master segment, as for the transfer of information between neighboring master segments within a node-to-segment approach. Within other approaches, the current transfer technique has to be modified including an algorithmic coupling with searching procedures.

We focus here on details of the transfer algorithm only. The full algorithm for contact problems based on an iterative Newton scheme, the structure of the corresponding tangent matrices and residuals and details of the finite element implementation are given in [3], [4], [1]. 


\section{Numerical example.}

In this section, we consider a special contact case, for which the application of the described scheme is absolutely necessary. An elastic strip AD, see Fig. 2 ( $E=2.1 \cdot 10^{4}, \nu=0.3$, thickness $h=0.5$, length $L=24$ ) is positioned at the beginning of a channel with width $a=13$. The channel itself is modeled by two rigid blocks $\mathbf{B}_{\mathbf{1}}$ and $\mathbf{B}_{\mathbf{2}}$. For contact the side part of the channel is modeled as a rigid surface described by an analytical function. The strip is loaded incrementally by a prescribed displacement $v$ at the center until it is inserted into the channel, see Fig. 3. The strip here is modeled with 24 linear solid-shell elements, see Harnau et al [1], and due to symmetry only one half of the system is modeled. The crucial point during the analysis is the sliding of a sharp corner C over the element boundaries 1, 2, 3, see Fig. 3. A load-displacement curve computed for the loading point is chosen as the representative parameter to study the effect of proposed approach. The following parameters are taken: load increment $v=0.0025$, penalty parameters $\varepsilon_{N}=2.1 \cdot 10^{5}, \varepsilon_{T}=2.1 \cdot 10^{5}$ and friction coefficient $\mu=0.2$. A straightforward analysis without the continuous transfer scheme leads to a disconvergence for frictional contact problems, see Fig. 4. For non frictional problem, though it is a converging case, the loaddisplacement curve contains jumps caused by the slave node passing from one master segment to another. The application of the continuous transfer scheme, however, allows to obtain the full force-displacement curve, even in the part when the strip is fully inserted into the channel, see the straight part of the curves in Fig. 4.

For comparison, the analysis is carried out also with different friction coefficients $\mu=0.1$ and $\mu=0.3$.

\section{Conclusions}

A continuous transfer algorithm for history variables based on the interpretation of covariant derivatives as a parallel translation is developed for frictional contact problems. The necessity of such a procedure is shown by a particular numerical example. The possibility to transfer other history variables in a similar fashion is also discussed for the example of updated sliding displacements; for further examples we refer to [5]. 


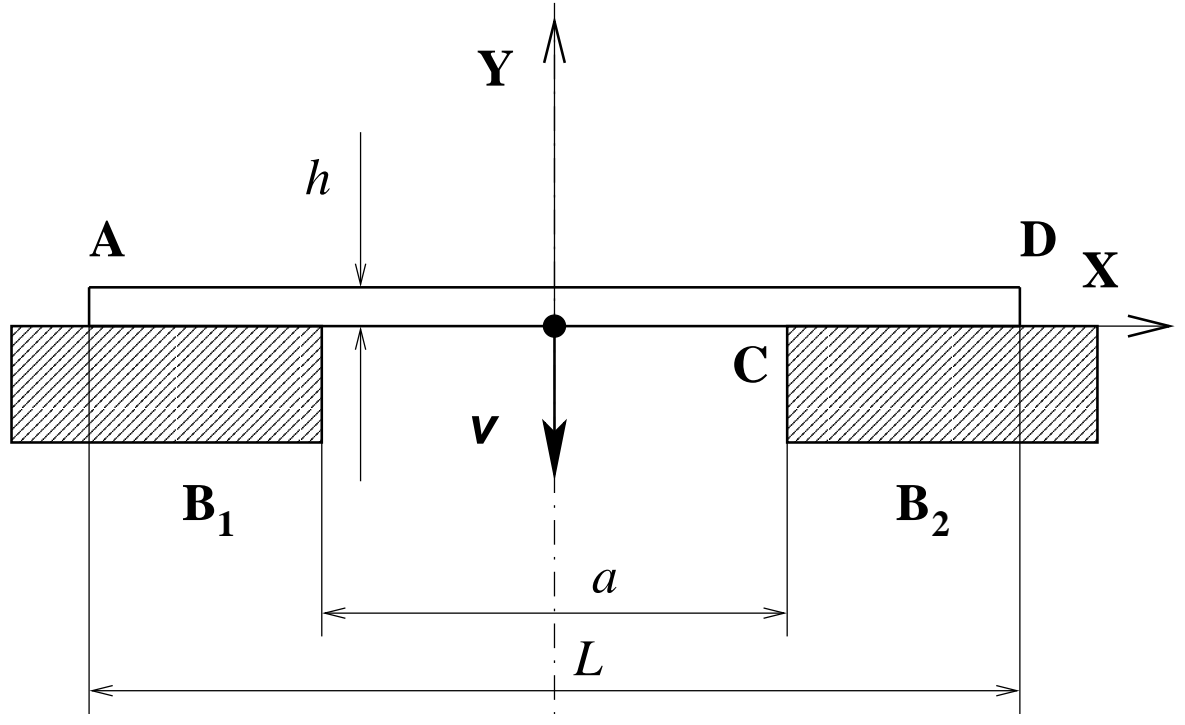

Figure 2: Drawing of an elastic strip into a channel with sharp corners. Definition of geometrical parameters.
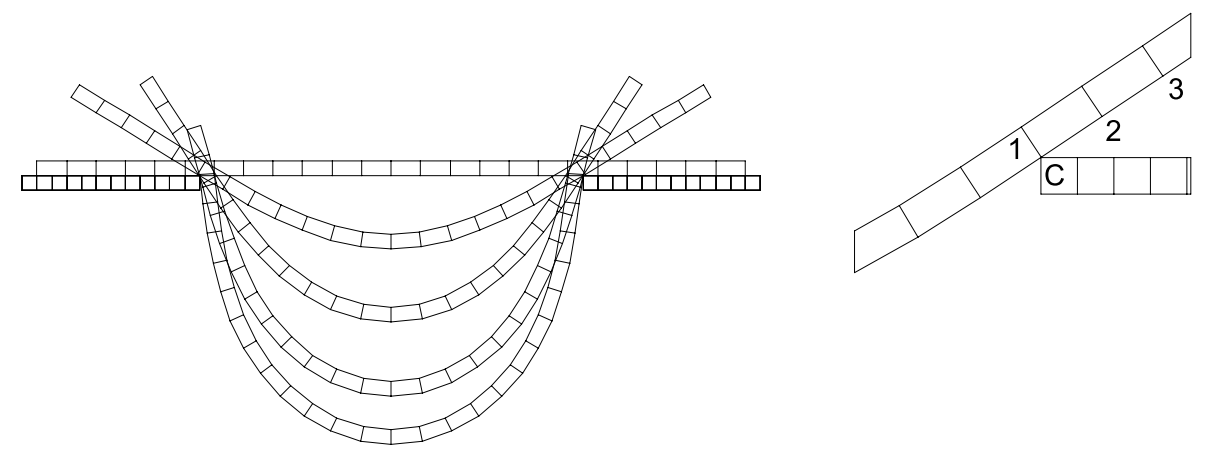

Figure 3: Sequence of deformations for the elastic strip. Nodes are sliding over the sharp corner C.

\section{References}

[1] Harnau M, Konyukhov A, Schweizerhof K (2005) Algorithmic aspects in large deformation contact analysis using "Solid-Shell" elements. Comput and Struct 83(21-22): 1804-1823.

[2] Jaiman RK, Jiao X, Geubelle PH, Loth E (2005) Assessment of conservative load transfer for fluid-solid interfaces with non-matching meshes. Int J Numer Meth Engng, 64:2014-2038.

[3] Konyukhov A, Schweizerhof K (2004) Contact formulation via a velocity description allowing efficiency improvements in frictionless contact analysis. Comput Mech 33:165-173. 


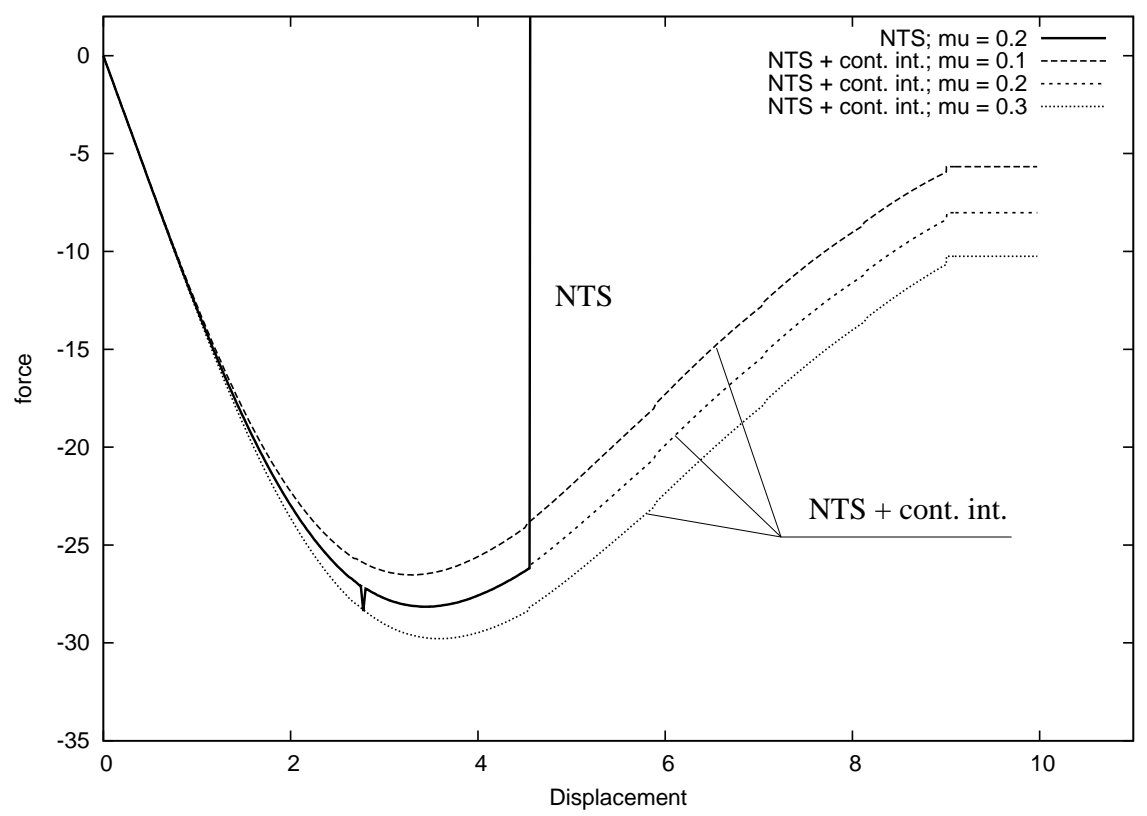

Figure 4: Drawing of an elastic strip over a sharp corner. Load-displacement curve. Frictional case - various friction coefficients. Straightforward NTS approach and continuous transfer scheme.

[4] Konyukhov A, Schweizerhof K (2005) Covariant description for frictional contact problems. Comput Mech 35:190-213.

[5] Konyukhov A, Schweizerhof K (2005) A special focus on 2D formulations for contact problems using a covariant description. Int J Numer Meth Engng 66:1432-1465.

[6] Laursen TA (2002) Computational Contact and Impact Mechanics. Fundamentals of Modeling Interfacial Phenomena in Nonlinear Finite Element Analysis. Springer: New-York, Heidelberg, Paris.

[7] Laursen TA, Simo JC (1993) A continuum-based finite element formulation for the implicit solution of multibody large deformation frictional contact problems. Int J Numer Meth in Engng 35:3451-3485.

[8] Puso MA, Laursen TA (2002) A 3D contact smoothing method using Gregory patches. Int J Numer Meth Engng, 54:1161-1194.

[9] Wriggers P, Krstulovic-Opara L, Korelc J (2001) Smooth $C^{1}$-interpolations for two-dimensional frictional contact problems. Int J Numer Meth Engng, 51:1469-1495.

[10] Wriggers P (2002) Computational Contact Mechanics, Wiley: Chichester. 